Urologe $2018 \cdot 57: 530-531$

https://doi.org/10.1007/s00120-018-0640-4

(C) Springer Medizin Verlag GmbH, ein Teil von Springer Nature 2018

CrossMark

\author{
M. Retz' $\cdot$ H. J. Bardenheuer ${ }^{2}$ \\ ' Urologische Klinik und Poliklinik, Technische Universität München, Klinikum rechts der Isar, München, \\ Deutschland \\ ${ }^{2}$ Klinik für Anästhesiologie, Überregionales Zentrum für Schmerztherapie und Palliativmedizin , \\ Universitäre Palliativmedizin Krhs. St. Vincentius, Universitätsklinikum Heidelberg, Heidelberg, \\ Deutschland
}

\title{
Supportive Therapie, „best supportive care" und Palliativmedizin
}

sammen und soll deren Anwendung in der täglichen Routine erleichtern.

Der zweite Beitrag befasst sich mit dem Nebenwirkungsmanagement der Immuntherapie. Immun-CheckpointInhibitoren gehören mittlerweile zu den Standardtherapien beim fortgeschrittenen bzw. metastasierten Urothel- und Nierenzellkarzinom. Insbesondere bei der Kombinationstherapie mit Nivolumab und Ipilimumab steigt nicht nur die Effektivität, sondern auch die $\mathrm{Ne}$ benwirkungsrate deutlich an. Prinzipiell können alle Organsysteme von immunvermittelten Nebenwirkungen betroffen sein. Der Artikel soll eine praktische Anleitung in der Diagnostik und Therapie immunvermittelter Nebenwirkungen liefern.

In einem weiteren Beitrag werden die wichtigsten onkologischen Notfälle zusammengefasst. Beginnend mit der febrilen Neutropenie über das Tumorlysesyndrom und die Paravasate stellt der Beitrag die klassischen chemotherapiebedingten Nebenwirkungen in umfangreicher Weise dar.

Seit März 2017 sind gesetzliche Regelungen geschaffen worden, um schwer kranken Patienten Cannabis als Medizin auf einem BtM-Rezept auf Kosten der Krankenkassen $\mathrm{zu}$ verordnen. Obwohl die Legalisierung der Verordnung auf Patientenseite mit einem „Hype“ in den Erwartungen beispielsweise an eine effektive Schmerzbekämpfung verbunden war, sind die wissenschaftlichen Erkenntnisse zur differenzierten Wirksamkeit von Cannabinoiden eher enttäuschend. Rasche et al. zeigen uns neben historischen Entwicklungen die Komplexität dieser Substanzgruppe auf. Die Autoren tragen zum Verständnis der Wirkprofile der zahlreichen Cannabinoidsubtypen bei und bieten mit ihrem kritischen Beitrag Hilfestellung für die ärztliche Entscheidung zu einem differenzierten Einsatz von Cannabis beim Patienten.

Spezialisierte ambulante Palliativversorgung (SAPV) ist - im Sozialgesetzbuch seit 2007 verankert - die Antwort der Politik auf den Patientenwunsch, die letzte Lebenszeit bei unheilbarer Erkrankung unter den professionellen Bedingungen einer palliativen ärztlichen und pflegerischen Betreuung in der vertrauten häuslichen Umgebung zu verbringen. Geist et al. skizzieren die Voraussetzungen zum Anspruch auf die gesetzlich garantierte Leistung und die vielfältigen Aufgaben der Palliativteams. Darüber hinaus liefert ihr Beitrag praktische Hilfe zur Umsetzung der SAPV-Verordnung im Bedarfsfall.

Frankenhauser et al. zeigen mit ihrem CME-Beitrag auf, dass interprofessionelle Symptomkontrolle am Lebensende Ausdruck verantwortlichen ärztlichen Handelns ist, wenn kurative Therapien in der Behandlung von Tumorerkrankungen nicht mehr wirksam sind. „Man lässt seinen Patienten nicht allein“ 
und ist ärztlich präsent mit einer hohen medizinischen, pharmakologischen, sozialen und spirituellen Professionalität.

\section{Korrespondenzadresse}

\section{Prof. Dr. M. Retz}

Urologische Klinik und Poliklinik, Technische

Universität München, Klinikum rechts der Isar Ismaningerstr. 22, 81675 München,

Deutschland

margitta.retz@tum.de

Prof. Dr., Prof. h. c. (RCH) H. J. Bardenheuer Klinik für Anästhesiologie, Überregionales Zentrum für Schmerztherapie und Palliativmedizin, Universitäre Palliativmedizin Krhs. St. Vincentius, Universitätsklinikum Heidelberg

Im Neuenheimer Feld 131, 69120 Heidelberg, Deutschland

Hubert.Bardenheuer@med.uni-heidelberg.de

Interessenkonflikt. M. Retz und H.J. Bardenheuer geben an, dass kein Interessenkonflikt besteht.

\section{Literatur}

1. Bauman JR, Temel JS (2014) The integration of early palliative care with oncology care: the time has come for a new tradition. J Natl Compr Canc Netw 12:1763-1771

\section{Lesen Sie Der Urologe online auf SpringerMedizin.de}

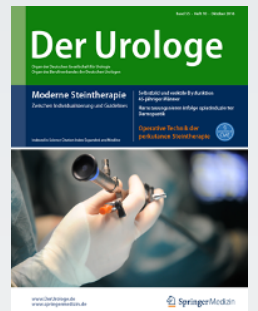

Auf SpringerMedizin.de erhalten Sie Zugang zu allen elektronisch verfügbaren Ausgaben und dem CME-Angebot Ihrer Zeitschrift - unabhängig davon, seit wann Sie Der Urologe abonniert haben. Außerdem können Sie die Zeitschrift mit dem E-Paper auch bequem auf Ihrem Tablet lesen.

So einfach erhalten Sie Zugang zum Online Archiv Registrieren Sie sich einmalig auf www.springermedizin.de/register

Geben Sie dabei Ihre Einheitliche Fortbildungsnummer (EFN) an.

- Ihr Benutzername entspricht Ihrer E-Mail-Adresse, Ihr Passwort können Sie frei wählen und später jederzeit unter „Mein Profil“ ändern.

- Falls Sie bereits ein (Print-) Abonnement bei uns haben, geben Sie bei der Registrierung die Lieferadresse Ihrer Zeitschrift an. Damit wird Ihr Abo-Zugang auf springermedizin.de freigeschaltet.
Sind Sie bereits bei SpringerMedizin.de registriert? Dann wird Ihr Zeitschriftenabonnement automatisch Ihrem Online-Nutzerkonto hinzugefügt. Sollten die Angaben Ihres Online-Accounts nicht eindeutig mit den Angaben Ihres Zeitschriften-Abonnements übereinstimmen, kann die Zuordnung nicht sicher erfolgen. In diesem Fall und bei allen anderen Fragen zum Online-Zugang kontaktieren Sie bitte unseren Kundenservice unter: Kundenservice@springermedizin.de

Telefonisch erreichen Sie die Hotline montags bis freitags von 9.00 bis 17.00 Uhr kostenfrei unter 0800-77 80777 sowie gebührenpflichtig aus dem Ausland unter $\hookleftarrow+4930884293600$ 